\title{
Determinants of plasma adropin associated with metabolic control and links to lipid and glucose homeostasis
}

\author{
Marietta Sayegh $^{1}$, Andrew Farquharson ${ }^{1}$, Graham Horgan ${ }^{2}$, Stuart R Gray ${ }^{3}$, Frank Thies ${ }^{1}$, \\ Viren Ranawana ${ }^{1}$ and Janice E. Drew ${ }^{1}$ \\ ${ }^{1}$ The Rowett Institute, \\ ${ }^{2}$ Biomathematics and Statistics Scotland, University of Aberdeen, Foresterhill, Aberdeen AB25 2ZD, UK and \\ ${ }^{3}$ Institute of Cardiovascular and Medical Sciences, University of Glasgow, GLASGOW, G12 8TA, UK
}

The peptide hormone, adropin, is altered by diet, reduced in obese individuals and associated with improved metabolic health linked to glucose and lipid metabolism, fat oxidation and vascular function ${ }^{(1,2)}$. Growing evidence identifies links between circulating adropin levels and regulation of blood glucose and lipids, implicating this hormone in metabolic diseases, such as diabetes and cardiovascular disease $(\mathrm{CVD})^{(2)}$. However, specific determinants influencing circulating adropin are not fully elucidated. This study aimed to identify determinants regulating plasma adropin levels and associations with metabolic control linked to lipid and glucose regulation.

Plasma adropin data measured using an ELISA kit (Cusabio, USA) was combined from three study cohorts: SIT (Sprint Interval Training) $(n=20)^{(3)}$, OGHH (Oats, Gut and Heart Health, clinicaltrials.gov Identifier ISRCTN11665494) $(n=40)$ and VegGI (Impact of Vegetables on acute Glycaemia and glycaemia induced CVD risk in women: metabolic and Inter-individual variations, researchregistry3117) $(n=31)$. Combined analysis of data from the three cohorts was conducted to identify correlates (Pearson correlations, Microsoft Excel) of adropin with age, sex, BMI and markers of metabolic health (fasted plasma lipids, glucose and insulin). Independent t-tests were calculated to assess differences in plasma adropin associated with age, sex and BMI. Principal Component Analysis (PCA) performed on plasma adropin, glucose and lipid data was used to conduct Partial Least Squares (PLS) analysis with anthropometric measures (age, sex, BMI) using SIMCA-P+ 12.0 software.

Combined analysis of data from the three cohorts $(n=83)$ identified that plasma adropin correlated: negatively with BMI $(-0.38$, $\mathrm{p}=0.00033)$, fasted plasma glucose $(-0.34, \mathrm{p}=0.00192)$ and positively with HDL $(0.47, \mathrm{p}=0.00001)$. However, PCA and PLS reveal complex patterns associated with plasma adropin linked to sex, BMI and metabolic markers. Females $(\mathrm{n}=59)$ with normal BMI, highest plasma HDL have higher plasma adropin compared to the rest of the cohort. Males $(n=24)$ with higher fasting glucose and insulin appear to have lower adropin, as do females who are older and, or, overweight or obese as classified by BMI.

Combined analysis of data from the three cohorts supports evidence linking adropin and metabolic control. However, future research on this potentially important metabolic target pathway must address the multiple determinants associated with plasma adropin. To date, reports present simplistic associations, without accounting for the multiple factors associated with plasma adropin levels. Sex differences, body composition and life stage, need to be considered to fully develop strategies to target adropin for pharmaceutical and lifestyle interventions in preventing obesity and improving metabolic health linked to lipid and glucose regulation.

1. Drew JE, Reichardt N, Williams LM et al. (2018) Sci Rep 8, 1.

2. Gao S, Stevens SR, Butler AA (2016) Expert Rev Endocrinol \& Metab 11, 239-241.

3. Gray SR, Aird TP, Farquharson AJ et al. (2018) APNM 43, 84-93. 